\title{
Personality disorder and alcohol treatment outcome: systematic review and meta-analysis
}

\author{
Giles M. Newton-Howes, James A. Foulds, Nicola H. Guy, Joseph M. Boden and Roger T. Mulder
}

\section{Background}

Personality disorders commonly coexist with alcohol use disorders (AUDS), but there is conflicting evidence on their association with treatment outcomes.

\section{Aims \\ To determine the size and direction of the association between personality disorder and the outcome of treatment for AUD. \\ Method \\ We conducted a systematic review and meta-analysis of randomised trials and longitudinal studies. \\ Results \\ Personality disorders were associated with more alcohol-related impairment at baseline and less retention in treatment. However, during follow-up people with a personality disorder showed a similar amount of}

improvement in alcohol outcomes to that of people without such disorder. Synthesis of evidence was hampered by variable outcome reporting and a low quality of evidence overall.

\section{Conclusions}

Current evidence suggests the pessimism about treatment outcomes for this group of patients may be unfounded. However, there is an urgent need for more consistent and better quality reporting of outcomes in future studies in this area.

\section{Declaration of interest}

None.

\section{Copyright and usage}

(c) The Royal College of Psychiatrists 2017.
The interplay between personality disorder and mental state disorder is increasingly recognised as needing clinical attention, with the former often significantly influencing the latter. There is a growing understanding that personality disorders are an important cause of morbidity worldwide, ${ }^{1}$ and that the disorder influences treatment outcomes for many other health conditions. ${ }^{2}$ Typically the coexistence of a personality disorder leads to worse outcomes in the treatment of mental state disorders, and this has been described for internalising disorders such as depression and eating disorders, ${ }^{3-5}$ as well as schizophrenia. ${ }^{6}$ It is not so clear, however, that a negative association exists between the outcomes of externalising mental state disorders and personality disorder. Nonetheless, personality has for some time been an area of research activity in externalising disorders, particularly alcohol use disorders (AUDs). Indeed, until the late 1960s alcoholism was considered to be a form of personality disorder. ${ }^{7}$ Population data from the USA suggest that $29 \%$ of those with a current AUD have a personality disorder. ${ }^{8}$ This figure is significantly higher than that in the general population, where the prevalence of personality disorder has been estimated at 2-6\% internationally. ${ }^{9}$ An even higher prevalence of personality disorder, particularly antisocial personality disorder (ASPD), has been reported in treatment-seeking samples of patients with AUD, ${ }^{10,11}$ with the prevalence of personality disorder occasionally reported as being over $50 \% .^{12}$ This range of prevalence data suggests both that personality disorder is clearly identifiable in patients with AUD and is more common in AUD than in the general population, but is not ubiquitous with the psychosocial problems in AUDs.

Given the high prevalence of personality disorder in patients with an AUD, and the evidence that personality disorder is relevant to treatment outcome in other mental state disorders, closer investigation of the role of personality disorder in treatment outcome for patients with AUD is needed. If it is the case that personality disorder is relevant, there is also a need to determine whether this is the case for all types of alcohol outcomes or just a subset. Although it has historically been believed that the presence of a coexisting personality disorder leads to worse treatment outcomes for patients with $\mathrm{AUD},{ }^{13}$ a recent review of predictors of AUD treatment outcome more generally did not find strong evidence that this is the case. ${ }^{14}$ However, this review identified only a small number of relevant studies, most of which focused on ASPD rather than other types of personality disorder. A narrative overview of the literature does not provide any clear indication of the association between AUD and personality disorder. We therefore aimed to perform a systematic review of the association between personality disorder status and alcohol treatment outcomes for patients with an AUD.

\section{Method}

Conduct and reporting of the review were designed to conform to guidelines for the Preferred Reporting Items for Systematic Reviews and Meta-analyses (PRISMA). ${ }^{15}$ The review protocol was registered with the International Prospective Register of Systematic Reviews (PROSPERO; registration CRD42015023972) prior to literature searching. A single amendment was made to the protocol to remove ambiguity following the literature search, but no change was made to the populations examined or the primary outcomes as predefined. The change specified the Addiction Severity Index as a measure of psychosocial functioning, ${ }^{16}$ the use of retention in treatment as a secondary outcome and provided clarity around quality assessment using the Newcastle-Ottawa Scale. ${ }^{17}$ The protocol was designed to identify studies reporting on the association between categorical personality disorders or dimensional personality traits and alcohol treatment outcomes in clinical trials. Only outcomes relating to categorical disorders are presented.

\section{Eligibility criteria}

Randomised controlled trials and longitudinal studies in which psychosocial and/or pharmacological treatments were provided 
for an AUD were considered for inclusion. Studies were required to have examined the association between personality disorder status and treatment outcome over at least 8 weeks. As the diagnostic criteria for AUD and personality disorder have evolved over time, any paper from 1980 onwards with clear diagnostic criteria for these two disorders was considered for inclusion. Studies were required to have been published in peer-reviewed journals subsequent to the introduction of DSM-III in 1980 because of the increased diagnostic rigour from this time, ${ }^{18}$ improving homogeneity of the populations studied. Dissertation abstracts and conference proceedings, and studies examining treatments for the personality disorder rather than AUD, were not considered for inclusion.

\section{Search strategy and selection criteria}

A broad search strategy was employed in line with prior personality disorder and mental state disorder systematic reviews, ${ }^{3,4}$ in order to minimise the risk of overlooking important research. Searches were undertaken using the Ovid platform in Medline, Cochrane, EMBASE and PsycINFO from 1980 until 24 November 2015. For personality disorder the search terms used were personality, personality disorder, antisocial borderline, schizoid. For AUDs the search terms were alcohol related disorder, alcohol dependence, alcohol abuse, alcohol addiction and alcoholi*. Results from each keyword within alcohol and personality clusters were combined with the Boolean classifier OR, and the two searches were then combined with the Boolean classifier AND. In addition, hand searches of articles cited by previous reviews and articles referenced in the papers in our final sample were conducted. Two authors (J.A.F. and G.M.N.-H.) independently screened the titles and abstracts of citations identified by the search strategy. Citations identified as potentially relevant were then compiled into a screened sample by consensus. The full text of these articles was then independently reviewed by two authors (N.H.G. and J.A.F. or G.M.N.-H.) to determine whether studies met inclusion criteria. Where two or more articles referred to the same study population, the article providing the most robust reporting of the systematic review's a priori primary outcomes was selected. Data from other articles pertaining to the same study sample were then used only to provide information about baseline patient data.

\section{Outcomes}

The primary alcohol outcomes were time to relapse and selfreported alcohol consumption (quantity and frequency). Two other outcomes that were not nominated a priori in the review protocol were also investigated, as it became apparent during the process of the review that they were commonly reported. These were retention in treatment and scores on the Addiction Severity Index. ${ }^{16}$

\section{Data collection and extraction}

A data extraction form was used to record key characteristics of each trial included in the final sample. Data were extracted independently by at least two authors (N.H.G. and G.M.N.-H. or J.A.F.). The data extracted were sample size, demographic characteristics of sample (age, gender), baseline AUD, type of interventions provided to study participants, treatment setting, study completion rate, alcohol outcomes and personality disorder diagnosis. All information was cross-checked by at least two authors conferring. Study authors were contacted with requests for further information on outcomes that were not clearly reported in published material. It was agreed a fourth review author (J.M.B. or R.T.M.) would moderate if consensus could not be reached in regard to inclusion or data extraction; however, this did not occur.

\section{Assessment of study bias}

Study quality and potential for bias was assessed using the Newcastle-Ottawa quality assessment scale. ${ }^{17}$ This is a widely used tool for assessing the quality of non-randomised studies, and was chosen in preference to tools that emphasise randomisation and blinding procedures, because treatment effects in clinical intervention studies were not of primary interest in this review. Reported outcomes were checked against published trial protocols. Disagreements about trial quality were resolved by discussion between at least two review authors.

\section{Synthesis of results}

For outcomes where results from at least three studies were available and reporting quality was adequate, heterogeneity was assessed using the $I^{2}$ measure. ${ }^{19}$ Random effects meta-analysis was then conducted using RevMan version 5.3 software. ${ }^{20}$ Where meta-analysis was not possible a narrative account of the quantitative findings of the included papers was used.

\section{Quality of evidence and risk of bias}

The GRADE approach was used to assess the quality of evidence for each clinical outcome across studies. ${ }^{21}$ This approach assesses the quality of a body of evidence for a particular outcome of interest by summarising data quality for that outcome across studies using a standardised heuristic. ${ }^{19}$ The GRADE method combines information about study conduct, consistency of outcome measures, generalisability and magnitude of effect size.

\section{Results}

In all, 22 studies were included in the review, comprising 4861 participants (Fig. 1). Of these studies, 21 were in English and 1 was in German. Of the 22 included studies, 9 primarily examined ASPD; $;^{22-30} 3$ examined borderline personality disorder (BPD); ${ }^{31-33}$ 1 investigated ASPD or BPD; ${ }^{34}$ and the remaining 9 studies reported on the presence of any personality disorder. ${ }^{35-43}$ The characteristics of these studies are shown in Table 1.

\section{Personality disorder prevalence}

Excluding one study that reported only on ASPD that developed before the onset of alcoholism, ${ }^{27}$ the prevalence of ASPD ranged from $15 \%$ to $41 \%$ (median $28 \%$ ) in the remaining eight ASPD studies; in the BPD studies, BPD prevalence ranged from $11 \%$ to $27 \%$ (median $18 \%$ ); and in studies reporting on any personality disorder, total prevalence ranged from $34 \%$ to $71 \%$ (median $55 \%)$.

\section{Baseline differences between patient groups}

Baseline differences between patients with and without personality disorder were assessed to examine whether any association found in outcomes was potentially explained by these differences. Baseline data were not presented in four papers. The reporting of baseline data in the remaining 18 papers was highly variable, and data were therefore summarised narratively rather than by meta-analysis or meta-regression. In the majority of studies alcohol consumption patterns at baseline did not differ between patients with and without a personality disorder, ${ }^{25,28,34,40,43}$ although three papers reported heavier alcohol consumption at 


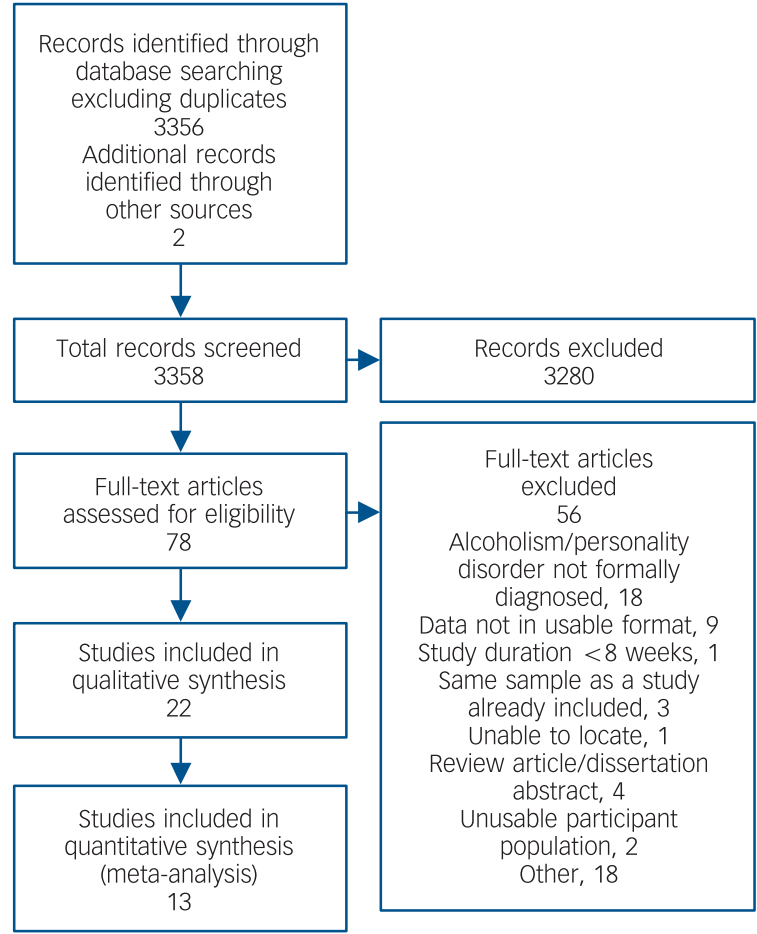

Fig. 1 Study search.

baseline among patients with ASPD. ${ }^{24,27,29}$ There was consistent evidence that patients with ASPD had earlier onset of alcohol use and alcohol problems. ${ }^{23,25,27,43}$ As expected, patients with ASPD also reported more legal problems, ${ }^{25,27,28,30}$ and more concurrent use of other substances. ${ }^{27,30,40,43}$ Several studies also reported an excess of physical, psychological and social problems among patients with ASPD, ${ }^{23,28}$ and among those with personality disorder more generally. ${ }^{40}$

\section{Primary alcohol outcomes}

A wide range of alcohol outcomes were reported, including the a priori outcomes (time to relapse and self-reported quantity and frequency of alcohol consumption). The reporting was, however, heterogeneous and variable in quality, meaning that meta-analyses were only able to include a minority of studies for each outcome. A lack of comparable outcome measures and incomplete statistical reporting were the primary problems.

Time to relapse

Three studies reported that the presence of personality disorder was associated with a significantly shorter time to first relapse. ${ }^{36,38,43}$ In a further study that examined time to readmission in a sample of male veterans, the presence of ASPD was not a significant predictor of readmission for alcohol-related problems. ${ }^{22}$

\section{Alcohol consumption}

No study reported alcohol consumption per drinking day or total consumption for those with personality disorder in general, or among patients other than those with cluster BPD. Only one of the four studies that reported on the number of standard drinks consumed per drinking day (DDD) identified personality disorder as a predictor of this alcohol outcome. In this study Kranzler et al reported that ASPD was associated with higher DDD during follow-up (7.3, s.d. =5.7) compared with those without ASPD $(4.9$, s.d. $=5.1) .{ }^{23}$ In a study of 229 patients Longabaugh et al found no overall difference in DDD between patients with and without ASPD during 18 months of follow-up, after adjusting for baseline DDD. ${ }^{24}$ Schuckit compared participants with primary ASPD (defined as people who had ASPD before the onset of alcohol or other substance problems) with other groups of participants; in this study the primary ASPD group had similar levels of DDD at follow-up $(15.7$, s.d. $=10.7)$ compared with those with primary alcoholism $(14.3$, s.d. $=10.8) .{ }^{27}$ Ralevski et al investigated the effect of ASPD or BPD in a sample of 254 predominantly male veterans. ${ }^{34}$ Patients with ASPD consumed slightly more alcohol in total at baseline compared with those without ASPD (10.9 drinks per day v. 9.6), whereas for BPD $v$. no BPD the figures were 10.3 v. 9.9 drinks respectively. At follow-up the average level of alcohol consumption was low $(<0.5$ drink per week) and heavy drinking was infrequent ( $<5 \%$ of days) across the whole sample. No difference in drinks per drinking day was reported according to the presence of ASPD or BPD.

Three studies $(n=875)$ were able to be included in a metaanalysis of DDD outcomes (Fig. 2). 23,27,34 Outcomes across these studies showed low heterogeneity $\left(I^{2}=20 \%\right)$. People with personality disorder had higher DDD at follow-up (pooled estimate of mean difference in DDD 2.15, 95\% CI 0.72-3.58). Two further studies reported findings for total alcohol consumption, with conflicting results. In a case-control study based on a cohort of patients receiving baclofen for alcohol dependence, Rolland et al compared participants with BPD and an age- and gender-matched control group. ${ }^{33}$ Patients with BPD drank substantially more per week than those without the disorder $(61.3$ drinks, s.d. $=49.7$ v. 14.7 drinks, s.d. $=24.1$ respectively) during follow-up; however, as patients were not matched on the basis of alcohol consumption at baseline, the follow-up findings are difficult to interpret. Conversely, in a prospective study of 351 patients admitted to in-patient detoxification in Iceland, ASPD was associated with heavier consumption at baseline but did not predict consumption at 1 year of follow-up. ${ }^{29}$ The majority of patients were abstinent at that point, suggesting that, like the rest of the sample, patients with ASPD generally had a good outcome.

\section{Percentage of days drinking}

As was the case for quantity of alcohol consumed, the percentage of drinking days (PDD) was not reported consistently, although the majority of papers found no association between personality disorder and PDD. In all, seven studies reported on the number or percentage of drinking days in the follow-up period. Schuckit found that patients with primary ASPD consumed alcohol on 191 days (s.d. $=141$ ) in the previous year compared with 170 days (s.d. = 138) for patients with primary alcoholism, a non-significant difference. ${ }^{27}$ Kranzler et al reported that both men and women with ASPD had more drinking days in 3 years of follow-up (202 days v. 157 days for men with and without ASPD respectively and 168 days $v .109$ days for women). ${ }^{23}$ However, this became non-significant after adjusting the model for confounders. Similarly, BPD, ASPD and personality disorder were not associated with higher PDD over a 12-month period irrespective of treatment population. ${ }^{25,32,40}$ In contrast to these studies Hunter-Reel et al reported a higher PDD in patients with cluster C personality disorder (but not other personality disorders) over a 6-month follow-up. ${ }^{37}$ However, analyses presented in this study were severely limited by low numbers of participants with each type of personality disorder. One further study also reported a higher PDD among people with ASPD $(21.7 \%$, s.d. $=34.0)$ compared with those without ASPD $(15.7 \%$, s.d. $=28.0)$ although this difference was not statistically significant. ${ }^{28}$ As shown in Fig. 2, three of the 


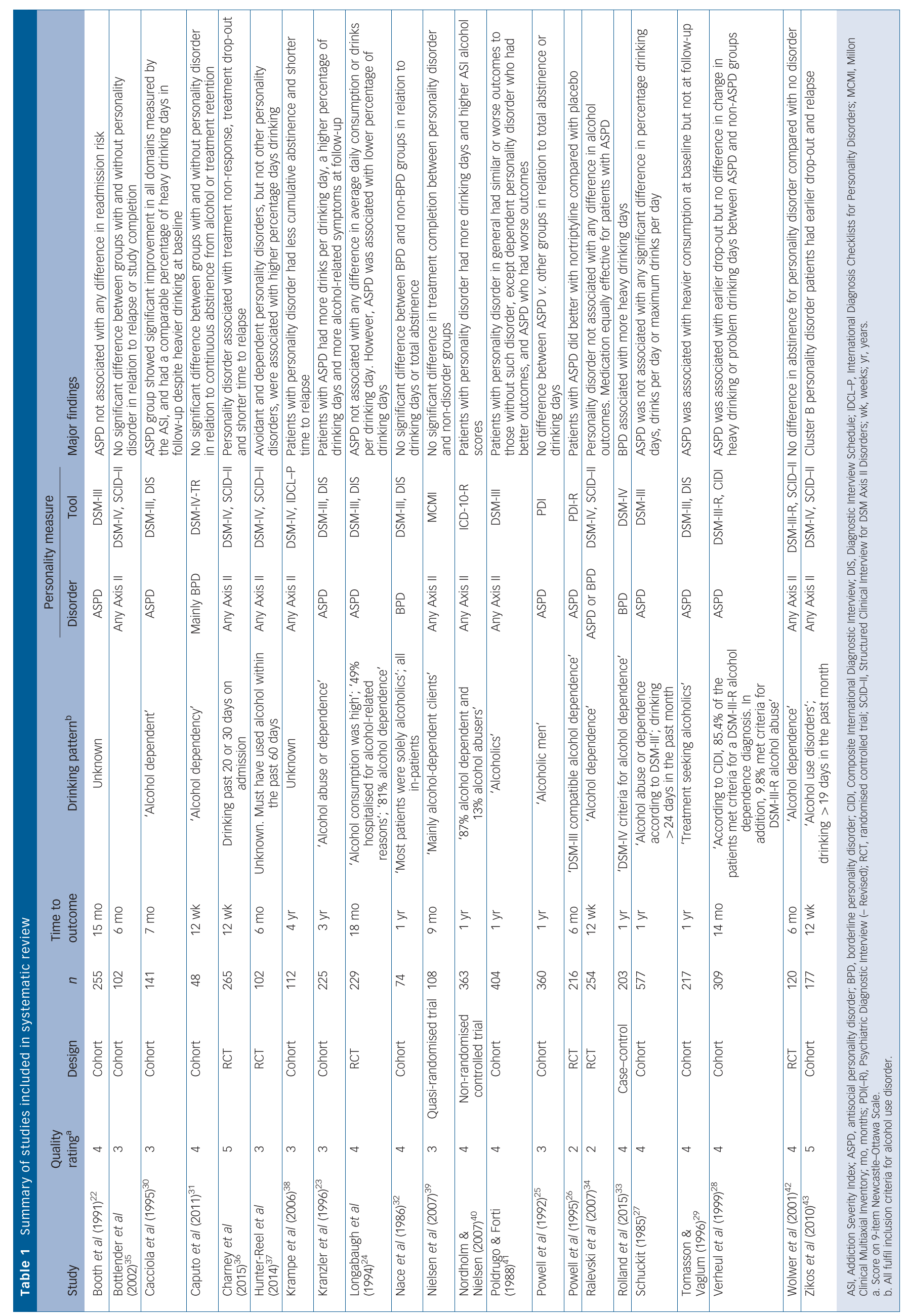




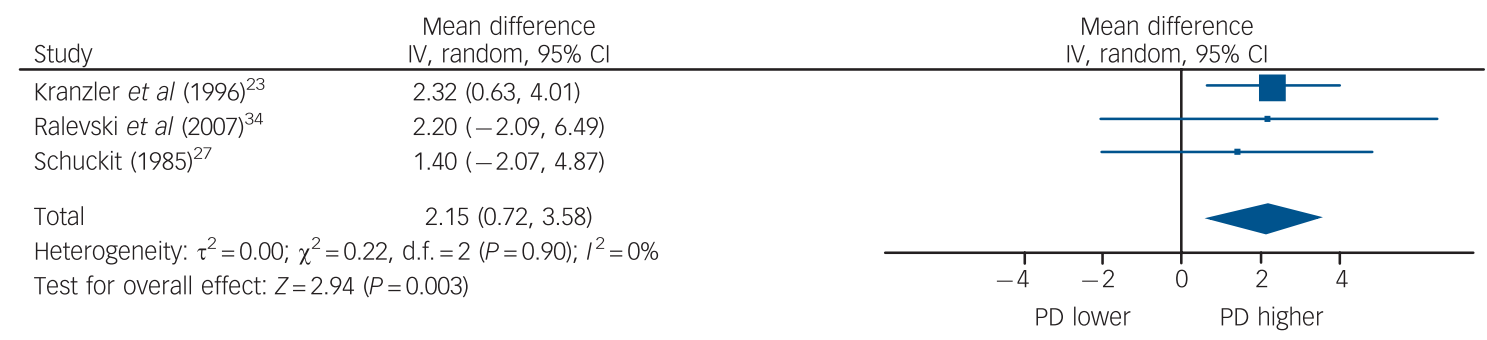

(b)

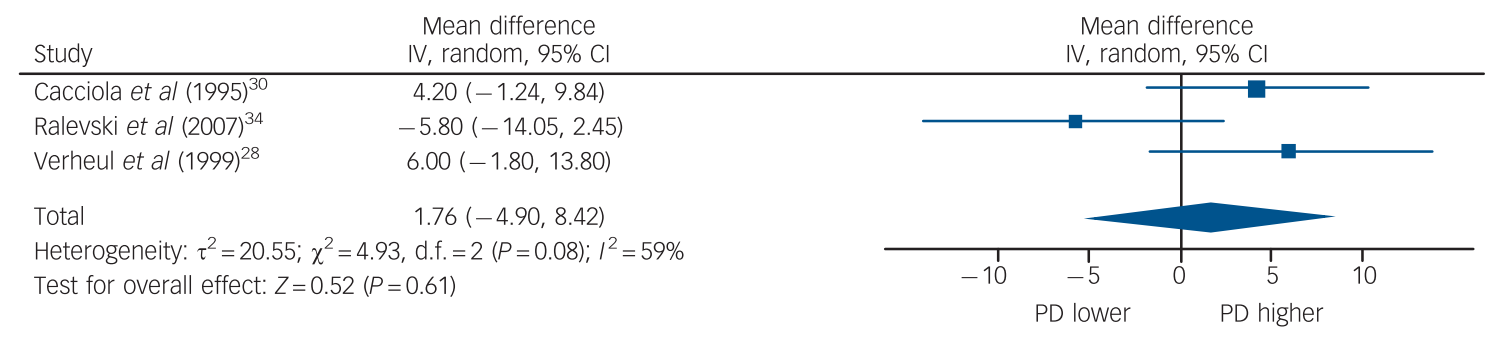

(c)

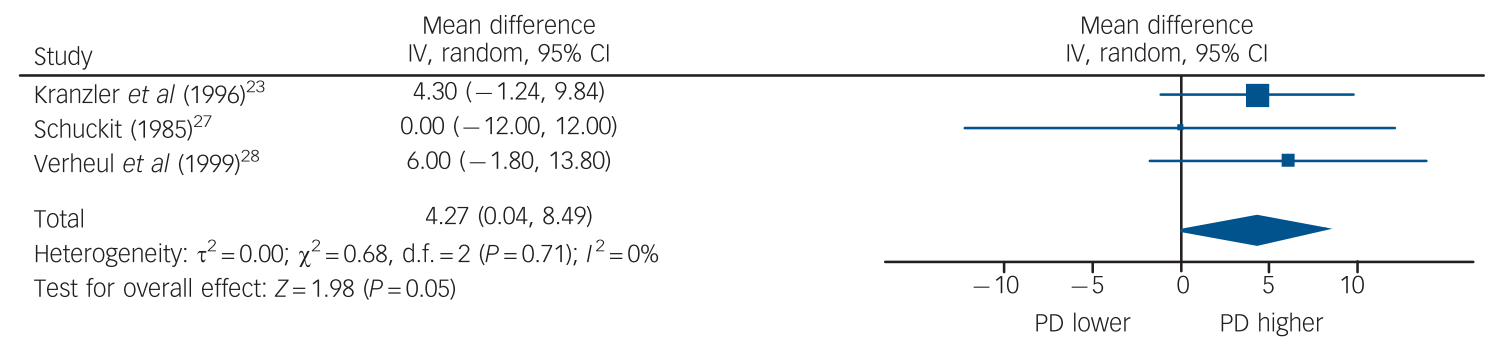

Fig. 2 Association between personality disorder status (PD) and primary alcohol outcomes: (a) drinks per drinking day; (b) percentage days heavy drinking; (c) percentage days drinking. For Ralevski et al comparison is between ASPD and no ASPD; Cacciola et al includes results from participants with primary cocaine dependence. ASPD, antisocial personality disorder.

above studies $(n=960)$ could be meta-analysed, ${ }^{23,27,28}$ all of which compared participants with and without ASPD. There was no heterogeneity in outcomes across studies $\left(I^{2}=0 \%\right)$. The PDD was estimated to be slightly higher in people with ASPD compared with those without that disorder (mean difference $4.27 \%, 95 \%$ CI 0.04-8.49).

\section{Percentage of days with heavy drinking}

Five studies reported on the percentage of heavy drinking days (PHDD) during follow-up, four of which showed similar improvement for patients with personality disorder compared with those without personality disorder. Verheul et al reported that participants with ASPD had higher PHDD (defined as at least five drinks) at baseline $(64.7 \%$, s.d. $=42.7)$ than the non-ASPD group $(55.7 \%$, s.d. $=36.3){ }^{28}$ At follow-up the ASPD group continued to have a higher PHDD $(21.7 \%$, s.d. $=34.0)$ than the non-ASPD group $(15.7 \%$, s.d. $=28.0)$ but the overall improvement was similar across both groups. Cacciola et al reported on PHDD (defined as drinking to intoxication) during follow-up over 7 months of treatment in a mixed sample of men with alcohol and/or cocaine dependency. ${ }^{30}$ In this study participants with childhood- or adult-onset ASPD drank to intoxication more often at baseline $35.1 \%$ for those with childhood-onset and $37.8 \%$ for those with adult-onset disorder) than those without ASPD $(20.7 \%)$. However, at follow-up the frequency of drinking to intoxication was $10.7 \%$ for the ASPD group and $6.5 \%$ for the non-ASPD group, suggesting that similar improvement was shown by both groups. A similar pattern of findings was reported by Nordholm \& Nielsen in a study of people attending out-patient alcohol treatment in Denmark. ${ }^{40}$ In this study PHDD (defined as more than three drinks per day) was $44.0 \%$ for those without personality disorder and $50.7 \%$ for those with personality disorder at baseline and $19.3 \%$ v. $23.0 \%$ respectively at follow-up, again implying a similar improvement regardless of personality disorder.

Ralevski et al reported that the PHDD at baseline (defined as days with five or more standard drinks) for participants with ASPD $(44.7 \%$, s.d. $=40.3)$ was similar to that of the remainder of the sample $(45.0 \%$, s.d. $=39.0){ }^{34}$ At baseline PHDD also did not differ according to whether BPD was present. At follow-up the PHDD was $14.3 \%$ (s.d. = 35.7) for those without ASPD and $8.5 \%$ (s.d. = 23.7) for those with the disorder. However, as the group of patients without ASPD also included some people with $\mathrm{BPD}$, these findings are difficult to interpret. Conversely, in the study by Rolland et al, people with BPD had a significantly higher PHDD (defined as an intake of at least $50 \mathrm{~g}$ of alcohol) at follow-up $(74.3 \%$, s.d. $=25.3)$ in comparison with those without that disorder $(41.7 \%$, s.d. $=33.3)$, although differences in drinking patterns between the two groups at baseline were not clearly reported. ${ }^{33}$ Three of the above studies, ${ }^{28,30,34}$ comprising 758 participants, reported data on the difference between ASPD and non-ASPD groups in a form that could be meta-analysed (Fig. 2). There was some variation within those studies in how heavy drinking was defined, and moderately high heterogeneity was present $\left(I^{2}=67 \%\right)$. There was no significant difference in PHDD between those with and without ASPD during follow-up (mean difference $1.76 \%$, 95\% CI -4.90 to 8.42 ).

\section{Secondary outcomes}

Retention in treatment

Eight studies reported on the odds of treatment retention for people with personality disorder compared with those without such disorder. Outcomes showed moderately high heterogeneity 


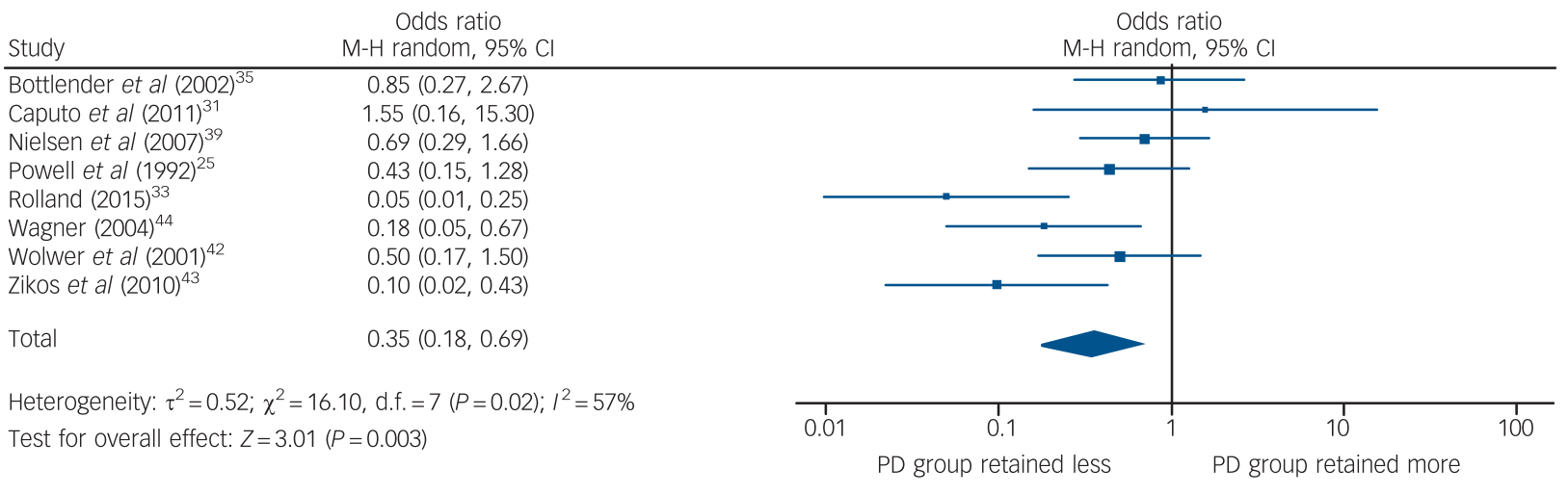

Fig. 3 Association between personality disorder status (PD) and treatment retention.

$\left(I^{2}=57 \%\right)$ so random effects meta-analysis was conducted. The pooled estimate of the odds of treatment retention was 0.35 (95\% CI 0.18-0.69), indicating that participants with personality disorder were more likely to withdraw before treatment completion (Fig. 3). Two further studies assessed the mean number of days in treatment, with one study reporting that people with ASPD had fewer days in treatment, ${ }^{28}$ and one reporting no difference between patients with ASPD, BPD or neither personality disorder. ${ }^{34}$

\section{Quality of evidence and risk of bias}

Table 2 outlines the quality and risks of bias across studies for each primary and secondary outcome, using a GRADE approach. ${ }^{45}$ It is notable that although the studies included in the systematic review comprised 4861 participants in total, meta-analyses of the primary outcomes stipulated in the review protocol comprised no more than three studies per outcome. This was due to the variable (but mainly poor) quality of statistical reporting and the diversity of alcohol outcomes employed in the studies selected. A number of studies did not report statistics in sufficient detail to allow calculation of an estimate of effect sizes and their variance, for example by reporting the mean of an outcome variable in the study sample but not its standard deviation. All included studies were of low or very low quality according to their scores on the Newcastle-Ottawa Scale. The overall quality of the evidence was very low, suggesting one high-quality study could significantly alter the findings of this review (see online Table DS1). The handling of missing data is a further issue with potential to bias the findings of individual studies. As noted, patients with personality disorders were more likely to withdraw from treatment, but in most cases studies reported findings for study completers. Few studies adopted robust statistical approaches to minimise bias arising from missing data. Therefore the findings reported in this review largely apply to individuals who were retained in follow-up, and they may not be generalisable to patients leaving the studies.

\section{Discussion}

Personality disorders are a common coexisting problem among patients presenting for treatment of an AUD. In our review up to two of every three patients had a comorbid personality disorder. Understanding the association between personality disorder and the outcome of treatment for an AUD therefore seems important. Although some evidence suggested that people with personality disorder were more impaired at the point of entry into treatment, with treatment they generally showed a similar amount of improvement to that of patients without personality disorder, assuming they remained in treatment (and their data were therefore analysed). This was the case both for specific alcohol-related outcomes and generic measures of psychosocial functioning (see online supplement DS1). In fact, in some areas improvements from baseline were at least as great as in those without personality disorders. This may be related to poorer baseline measures and subsequently greater capacity for gain with successful alcohol treatment. A standard difficulty with research examining personality disorder in mental state disorder is the stability of the personality disorder diagnosis over time with the treatment of the mental state disorder. This difficulty is well described for internalising disorders. ${ }^{46}$ This systematic review identifies similar problems existing for externalising disorders such as AUD. Although the short time frame may be considered problematic, research examining change in alcohol use in patients with personality disorder suggest much of the change is early, ${ }^{47}$ albeit sustained positive change is most clinically relevant. There was, however, some evidence that people with a personality disorder are less likely to complete treatment, an important proxy marker for longer-term success. ${ }^{48}$ This suggests that modifications to treatment interventions aimed at retaining patients with personality disorder in treatment might be worthwhile. Evidence-based interventions that improve retention in addictions treatment in general, ${ }^{49}$ and specifically in individuals with personality disorder, ${ }^{50}$ are likely to add value to overall outcome.

Frustratingly, efforts to synthesise data identified in this review were hampered by several key methodological limitations of the included papers. First, the overall quality of data reporting was poor; further, the quality of evidence across studies for each of the primary outcomes was similarly poor, partly because evidence was largely based on observational data. Marked variation in the alcohol outcomes reported by different studies meant that each meta-analysis included only a few studies. Finally, no attempt was made to meta-analyse the change in alcohol consumption patterns from baseline to study completion. This was because some studies that reported these measures adequately for the whole sample did not give a breakdown according to personality disorder, whereas some studies included in the meta-analyses presented in Fig. 2 did not adequately report baseline data. For these reasons we are cautious about suggesting that our findings truly represent the relationship between AUD outcomes and personality disorder in general, or for specific personality disorder clusters or subtypes. ${ }^{51}$ Personality disorders are a heterogeneous group in terms of patterns of behaviour, cognition and subjective distress. We have followed the general personality disorder approach in line with the likely changes to the ICD, ${ }^{52}$ as this increases the power of our findings and is forward-facing, 


\begin{tabular}{|c|c|c|c|c|}
\hline Outcome & $\begin{array}{c}\text { Total } \\
\text { participants, } n\end{array}$ & Unadjusted alcohol outcomes at follow-up & $\begin{array}{l}\text { Quality } \\
\text { of evidence }\end{array}$ & Comments $^{b}$ \\
\hline \multicolumn{5}{|l|}{ Primary outcomes } \\
\hline Time to relapse & 809 & $\begin{array}{l}\text { Personality disorder was associated with shorter } \\
\text { time to relapse in } 3 / 4 \text { studies }\end{array}$ & very low & $\begin{array}{l}\text { Incomplete reporting in some studies. } \\
\text { Difficult to quantify magnitude of any effect }\end{array}$ \\
\hline $\begin{array}{l}\text { Percentage of days } \\
\text { drinking }\end{array}$ & 1930 & $\begin{array}{l}\text { PDD slightly higher in participants with ASPD } \\
\text { V. those without (mean difference } 4.27 \% \text {, } \\
95 \% \mathrm{Cl} 0.04 \text { to } 8.49 \text { ) }\end{array}$ & very low & $\begin{array}{l}\text { Incomplete reporting in some studies but } \\
\text { good consistency across studies; most studies } \\
\text { reported findings only for completers group; } \\
\text { only } 3 \text { studies meta-analysed }\end{array}$ \\
\hline $\begin{array}{l}\text { Percentage of days with } \\
\text { heavy drinking }\end{array}$ & 1394 & $\begin{array}{l}\text { No significant difference in PHDD between } \\
\text { participants with and without ASPD during } \\
\text { follow up (mean difference } 1.76 \% \text {, } \\
95 \% \mathrm{Cl}-4.90 \text { to } 8.42 \text { ). }\end{array}$ & very low & $\begin{array}{l}\text { Incomplete reporting in some studies; most } \\
\text { studies reported findings only for completers } \\
\text { group; only } 3 \text { studies meta-analysed }\end{array}$ \\
\hline $\begin{array}{l}\text { Alcohol consumption: } \\
\text { drinks per drinking day }\end{array}$ & 1285 & $\begin{array}{l}\text { Participants with personality disorder had higher } \\
\text { DDD at follow-up (mean difference in DDD } 2.15 \text {, } \\
95 \% \mathrm{Cl} 0.72 \text { to } 3.58 \text { ) }\end{array}$ & very low & $\begin{array}{l}\text { Incomplete reporting; lack of agreement } \\
\text { between studies. Most studies reported } \\
\text { findings only for completers group; } \\
\text { only } 3 \text { studies meta-analysed }\end{array}$ \\
\hline \multicolumn{5}{|l|}{ Other outcomes } \\
\hline Treatment retention & 474 & $\begin{array}{l}\text { Personality disorder was associated with lower } \\
\text { odds of treatment retention }(\mathrm{OR}=0.35,95 \% \mathrm{Cl} \\
0.15 \text { to } 0.69)\end{array}$ & Low & Modest consistency between studies \\
\hline $\begin{array}{l}\text { Alcohol-related } \\
\text { psychosocial impairment }\end{array}$ & 1417 & $\begin{array}{l}\text { Personality disorder was associated with more } \\
\text { impairment at baseline but no difference in the } \\
\text { amount of improvement during treatment }\end{array}$ & very low & $\begin{array}{l}\text { Moderate consistency across studies } \\
\text { in spite of variable outcome measures used }\end{array}$ \\
\hline
\end{tabular}

although these concerns remain. This is despite this being the most comprehensive review of this literature to date.

Although personality disorders are highly prevalent in people with alcoholism, and are an important prognostic indicator for mental disorders in general, ${ }^{1,43}$ few previous reviews have attempted to investigate the association between personality disorder and treatment outcome in AUD. However, in a review of prognostic indicators in alcoholism more generally, Adamson et al found limited evidence that personality disorder is a predictor of altered treatment outcomes. ${ }^{54}$ It is also noteworthy that some major clinical trials in the alcohol field, such as Project MATCH and COMBINE, ${ }^{55,56}$ are absent from the set of studies included in our review. In the case of the COMBINE study this may be because Structured Clinical Interview for DSM Axis II Disorders modules were not part of the baseline assessment. ${ }^{57}$ For Project $\mathrm{MATCH}$, the study presented detailed data on ASPD $\times$ treatment interaction effects but without apparent consideration of any main effects of ASPD on alcohol outcomes. ${ }^{58}$ This suggests the opportunity to investigate the prognostic significance of personality disorder may not have been fully exploited in either of these large and important studies. It is possible such analyses could still significantly add to our understanding of the association between personality disorder and AUD.

\section{Study limitations}

The conclusions of this review must be viewed as tentative because of a number of limitations. The included studies were mainly small to medium in size and the quality of evidence was low for reasons already discussed. Statistical reporting was generally poor. Furthermore, our inferences about the association between personality disorder and treatment outcome are largely based on unadjusted observational data for participants who were retained in treatment. No study used modern statistical approaches for handling missing data, and the higher withdrawal rates among participants with personality disorder meant that analysing only the completer group is likely to have introduced bias. It is also likely that the effect of personality disorder on alcohol treatment outcomes is confounded by other covariates such as age, gender, severity of alcohol problems and other psychopathological disorder at baseline. However, few studies presented adjusted outcomes. Therefore future studies investigating this area should carefully consider the role of these and other covariates. It is also possible that blinding to status could have influenced outcome, as clinicians can have a bias towards patients with personality disorder; ${ }^{59}$ however, post hoc sensitivity analysis for this was not possible as no study provided information on blinded follow-up assessment and baseline personality status.

\section{Future research}

The findings from this review also speak to an urgent need to achieve better harmonisation of outcomes across studies in the alcohol field. In other areas of psychiatry such as mood disorders there tends to be much less variability in outcome reporting. For example, in a recent review of mood outcomes among people with AUD ${ }^{60}$ we reported that the majority of studies used the Hamilton Rating Scale for Depression (HRSD). Thus, in spite of concerns about the validity of scales such as the HRSD, ${ }^{61}$ consistent use at least makes quantitative synthesis of data across studies straightforward. Given that results from individual studies are often unreliable, consensus guidelines on the reporting of alcohol clinical trials are needed in order to help pool evidence from multiple studies.

A further development likely to influence this area is the rapid evolution in personality disorder classification, with a shift towards a more dimensional approach. ${ }^{52}$ This shift will entail a new era of studies investigating the association between dimensional personality domains and treatment outcomes in alcoholism and other substance use disorders. The new era presents a much-needed opportunity to improve the quality of evidence in this area. 
Giles M. Newton-Howes, MRCPsych, Department of Psychological Medicine, University of Otago, Wellington; James A. Foulds, FRANZCP, Department of Psychological Medicine, University of Otago, Christchurch; Nicola H. Guy, MMedSci, Department of Psychological Medicine, University of Otago, Wellington; Joseph M. Boden, PhD, Christchurch Health and Development Study, Roger T. Mulder, FRANZCP, Department of Psychological Medicine, University of Otago, Christchurch, New Zealand

Correspondence: Giles Newton-Howes, Department of Psychological Medicine, University of Otago, 23 Mein Street, Wellington, New Zealand. Email: Giles.newton-howes@otago.ac.nz

First received 18 Oct 2016, final revision 14 Dec 2016, accepted 26 Jan 2017

\section{References}

1 Tyrer $\mathrm{P}$, Mulder R, Crawford M, Newton-Howes G, Simonsen E, Ndetei D, et al. Personality disorder: a new global perspective. World Psychiatry 2010 9: $56-60$.

2 Tyrer P, Reed GM, Crawford MJ. Classification, assessment, prevalence, and effect of personality disorder. Lancet 2015; 385: 717-26.

3 Newton-Howes G, Tyrer P, Johnson T. Personality disorder and the outcome of depression: meta-analysis of published studies. Br J Psychiatry 2006; 188 13-20.

4 Newton-Howes G, Tyrer P, Johnson T, Mulder R, Kool S, Dekker J, et al. Influence of personality on the outcome of treatment in depression: systematic review and meta-analysis. J Pers Disord 2014; 28: 577-93.

5 Steinhausen HC. The outcome of anorexia nervosa in the 20th century. Am J Psychiatry 2002; 159: 1284-93.

6 Newton-Howes G, Marsh R. Personality dysfunction and social functioning in schizophrenia. Personal Ment Health 2013; 7: 114-21.

7 Sellman JD, Foulds JA, Adamson SJ, Todd FC, Deering DE. DSM-5 alcoholism: a 60-year perspective. Aust NZ J Psychiatry 2014; 48: 507-11.

8 Grant BF, Stinson FS, Dawson DA, Chou SP, Ruan WJ, Pickering RP. Co-occurrence of 12-month alcohol and drug use disorders and personality disorders in the United States: results from the National Epidemiologic Survey on Alcohol and Related Conditions. Arch Gen Psychiatry 2004; 61: 361-8.

9 Huang Y, Kotov R, De Girolamo G, Preti A, Angermeyer M, Benjet C, et al. DSM-IV personality disorders in the WHO World Mental Health Surveys. Br J Psychiatry 2009; 195: 46-53.

10 Arias F, Szerman N, Vega P, Mesias B, Basurte I, Morant C, et al. Alcohol abuse or dependence and other psychiatric disorders. Madrid study on the prevalence of dual pathology. Ment Health Subst Use 2013; 6: 339-50.

11 Ross HE, Glaser FB, Germanson T. The prevalence of psychiatric disorders in patients with alcohol and other drug problems. Arch Gen Psychiatry 1988; 45: 1023-31.

12 Bowden-Jones O, Iqbal MZ, Tyrer $\mathrm{P}$, Seivewright N, Cooper S, Judd A, et al. Prevalence of personality disorder in alcohol and drug services and associated comorbidity. Addiction 2004; 99: 1306-14.

13 Rounsaville BJ, Dolinsky ZS, Babor TF, Meyer RE. Psychopathology as a predictor of treatment outcome in alcoholics. Arch Gen Psychiatry 1987; 44: 505-13.

14 Adamson SJ, Sellman JD, Frampton CM. Patient predictors of alcohol treatment outcome: a systematic review. J Subst Abuse Treat 2009; 36: 75-86.

15 Moher D, Liberati A, Tetzlaff J, Altman DG. Preferred reporting items for systematic reviews and meta-analyses: the PRISMA statement. Ann Intern Med 2009; 151: 264-9.

16 McLellan AT, Luborsky L, Woody GE, O'Brien CP. An improved diagnostic evaluation instrument for substance abuse patients: the Addiction Severity Index. J Nerv Ment Dis 1980; 168: 26-33.

17 Wells G, Shea B, O'Connell D, Peterson J, Welch V, Losos M, et al. The Newcastle-Ottawa Scale (NOS) for assessing the quality of nonrandomised studies in meta-analyses. The Ottawa Hospital Research Institute, 2000.

18 American Psychiatric Association. Diagnostic and Statistical Manual of Psychiatric Disorders (3rd edn) (DSM-III). APA, 1980

19 Higgins JP, Green S. Cochrane Handbook for Systematic Reviews of Interventions. Wiley, 2008.

20 Cochrane Collaboration. Review Manager (RevMan) 5.0. Nordic Cochrane Centre, 2008.

21 Guyatt $\mathrm{GH}$, Oxman $A D$, Vist $G E$, Kunz $R$, Falck-Ytter $Y$, Alonso-Coello $P$, et al. GRADE: an emerging consensus on rating quality of evidence and strength of recommendations. BMJ 2008; 336: 924-6.
22 Booth BM, Yates WR, Petty F, Brown K. Patient factors predicting early alcohol-related readmissions for alcoholics: role of alcoholism severity and psychiatric co-morbidity. J Stud Alcohol 1991; 52: 37-43.

23 Kranzler HR, Del Boca FK, Rounsaville BJ. Comorbid psychiatric diagnosis predicts three-year outcomes in alcoholics: a posttreatment natural history study. J Stud Alcohol 1996; 57: 619-26.

24 Longabaugh R, Rubin A, Malloy P, Beattie M, Clifford PR, Noel N. Drinking outcomes of alcohol abusers diagnosed as antisocial personality disorder. Alcoholism 1994; 18: 778-85.

25 Powell BJ, Penick EC, Nickel EJ, Liskow BI, Riesenmy KD, Campion SL, et al. Outcomes of co-morbid alcoholic men: a 1-year follow-up. Alcoholism 1992; 16: $131-8$.

26 Powell BJ, Campbell JL, Landon JF, Liskow BI, Thomas HM, Nickel EJ, et al. A double-blind, placebo-controlled study of nortriptyline and bromocriptine in male alcoholics subtyped by comorbid psychiatric disorders. Alcoholism 1995; 19: 462-8.

27 Schuckit MA. The clinical implications of primary diagnostic groups among alcoholics. Arch Gen Psychiatry 1985; 42: 1043-9.

28 Verheul R, Van Den Brink W, Koeter MWJ, Hartgers C. Antisocial alcoholic patients show as much improvement at 14-month follow-up as nonantisocial alcoholic patients. Am J Addict 1999; 8: 24-33.

29 Tomasson K, Vaglum P. Psychopathology and alcohol consumption among treatment-seeking alcoholics: a prospective study. Addiction 1996; 91: 1019-30.

30 Cacciola JS, Alterman Al, Rutherford MJ, Snider EC. Treatment response of antisocial substance abusers. J Nerv Ment Dis 1995; 183: 166-71.

31 Caputo F, Francini S, Brambilla R, Vigna-Taglianti F, Stoppo M, Del Re A, et al. Sodium oxybate in maintaining alcohol abstinence in alcoholic patients with and without psychiatric comorbidity. Eur Neuropsychopharmacol 2011; 21: 450-6.

32 Nace EP, Saxon JJ, Shore N. Borderline personality disorder and alcoholism treatment: a one-year follow-up study. J Stud Alcohol 1986; 47: 196-200.

33 Rolland B, Valin T, Langlois C, Auffret M, Gautier S, Deheul S, et al. Safety and drinking outcomes among patients with comorbid alcohol dependence and borderline personality disorder treated with high-dose baclofen: a comparative cohort study. Int Clin Psychopharmacol 2015; 30: 49-53.

34 Ralevski E, Ball S, Nich C, Limoncelli D, Petrakis I. The impact of personality disorders on alcohol-use outcomes in a pharmacotherapy trial for alcohol dependence and comorbid Axis I disorders. Am J Addict 2007; 16: 443-9.

35 Bottlender M, Bottlender R, Scharfenberg CD, Soyka M. Effectiveness of outpatient treatment for alcoholism-impact of personality disorders on course of treatment [in German]. Psychother Psychosom Med Psychol 2002; 53: 384-9.

36 Charney DA, Heath LM, Zikos E, Palacios-Boix J, Gill KJ. Poorer drinking outcomes with citalopram treatment for alcohol dependence: a randomized, double-blind, placebo-controlled trial. Alcoholism 2015; 39: 1756-65.

37 Hunter-Reel D, Epstein EE, McCrady B, Eddie D. Personality disorders and the prediction of alcohol use outcomes for women: dimensional versus categorical classification. Addict Res Theory 2014; 22: 176-80.

38 Krampe H, Wagner T, Stawicki S, Bartels C, Aust C, Kroener-Herwig B, et al. Personality disorder and chronicity of addiction as independent outcome predictors in alcoholism treatment. Psychiatr Serv 2006; 57: 708-12.

39 Nielsen P, Rojskjaer S, Hesse M. Personality-guided treatment for alcohol dependence: a quasi-randomized experiment. Am J Addict 2007; 16: 357-64.

40 Nordholm D, Nielsen B. Personality disorders among Danish alcoholics attending outpatient treatment. Eur Addict Res 2007; 13: 222-9.

41 Poldrugo F, Forti B. Personality disorders and alcoholism treatment outcome Drug Alcohol Depend 1988; 21: 171-6.

42 Wolwer W, Burtscheidt W, Redner C, Schwarz R, Gaebel W. Out-patient behaviour therapy in alcoholism: impact of personality disorders and cognitive impairments. Acta Psychiatr Scand 2001; 103: 30-7.

43 Zikos E, Gill KJ, Charney DA. Personality disorders among alcoholic outpatients: Prevalence and course in treatment. Can J Psychiatry 2010; 55: 65-73.

44 Wagner T, Krampe H, Stawicki S, Reinhold J, Jahn H, Mahlke K, et al. Substantial decrease of psychiatric comorbidity in chronic alcoholics upon integrated outpatient treatment - results of a prospective study. J Psychiatr Res 2004; 38: 619-35.

45 Oxman $A D$, Group GW. Grading quality of evidence and strength of recommendations. BMJ 2004; 328: 1490-4.

46 Ferro $\mathrm{T}$, Klein DN, Schwartz JE, Kasch KL, Leader JB. 30-month stability of personality disorder diagnoses in depressed outpatients. Am J Psychiatry 1998; 155: 653-9. 
47 Foulds J, Mulder RT, Newton-Howes G, Adamson SJ, Boden JM, Sellman JD, et al. Personality predictors of drinking outcomes in depressed alcoholdependent patients. Alcohol Alcohol 2016; 51: 296-301.

48 Newton-Howes G, Stanley J. Patient characteristics and predictors of completion in residential treatment for substance use disorders. Psychiatr Bull 2015; 39: 221-7.

49 Dutra L, Stathopoulou G, Basden S, Leyro T, Powers M, Otto M. A metaanalytic review of psychosocial interventions for substance use disorders. Am J Psychiatry 2008; 165: 179-87.

50 Tyrer P. Nidotherapy: a new approach to the treatment of personality disorder. Acta Psychiatr Scand 2002; 105: 469-71.

51 Balshem $H$, Helfand $M$, Schunemann $H J$, Oxman AD, Kunz R, Brozek J, et al GRADE guidelines: 3. Rating the quality of evidence. J Clin Epidemiol 2011; 64: 401-6.

52 Tyrer $\mathrm{P}$, Crawford $\mathrm{M}$, Mulder R, Blashfield R, Farnam A, Fossati $\mathrm{A}$, et al. The rationale for the reclassification of personality disorder in the 11th revision of the international classification of diseases (ICD-11). Personal Ment Health 2011; 5: 246-59.

53 Newton-Howes G, Tyrer P, North B, Yang M. The prevalence of personality disorder in schizophrenia and psychotic disorders: systematic review of rates and explanatory modelling. Psychol Med 2008; 38: 1075-82.

54 Adamson SJ, Sellman JD, Frampton CM. Patient predictors of alcohol treatment outcome: a systematic review. J Subst Abuse Treat 2009; 36: 75-86.
55 Allen JP, Mattson ME, Miller WR, Tonigan JS, Connors GJ, Rychtarik RG, et al. Matching alcoholism treatments to client heterogeneity: project MATCH posttreatment drinking outcomes. J Stud Alcohol 1997; 58: 7-29.

56 Anton RF, O'Malley SS, Ciraulo DA, Cisler RA, Couper D, Donovan DM, et al. Combined pharmacotherapies and behavioral interventions for alcohol dependence: the COMBINE Study: a randomized controlled trial. JAMA 2006; 295: 2003-17.

57 COMBINE Study Research Group. Testing combined pharmacotherapies and behavioral interventions in alcohol dependence: rationale and methods. Alcohol Clin Exp Res 2003; 27: 1107-22.

58 Allen J, Anton RF, Babor TF, Carbonari J. Project MATCH secondary a priori hypotheses. Addiction 1997; 92: 1671-98.

59 Newton-Howes G, Weaver T, Tyrer P. Attitudes of staff towards patients with personality disorder in community mental health teams. Aust NZ J Psychiatry 2008; 42: 572-7

60 Foulds JA, Adamson SJ, Boden JM, Williman JA, Mulder RT. Depression in patients with alcohol use disorders: systematic review and meta-analysis of outcomes for independent and substance-induced disorders. J Affect Disord 2015; 185: 47-59.

61 Bagby RM, Ryder AG, Schuller DR, Marshall MB. The Hamilton Depression Rating Scale: has the gold standard become a lead weight? Am J Psychiatry 2004; 161: 2163-77.

\section{psychiatry in music}

\section{Corporal Clegg}

\author{
Russell Hinton
}

'Corporal Clegg', written by Roger Waters, appears on the second Pink Floyd album, the psychedelic space rock, A Saucerful of Secrets, released in 1968. The album represents something of a crossroads for the band with it being the first to feature David Gilmour and the last to feature Syd Barrett, whose declining mental health would ultimately result in him being forced to leave the band.

The song is about a soldier who has developed post-traumatic stress disorder (PTSD) as a result of his combat experiences. It is one of the first Pink Floyd songs to incorporate themes of mental illness and anti-war sentiment, which foreshadows much of their later work such as 'Brain Damage', 'Shine On You Crazy Diamond', 'Wish You Were Here', 'Comfortably Numb', 'Another Brick In The Wall', 'In The Flesh', 'Goodbye Blue Sky', and 'Bring The Boys Back Home'.

On first listening the track sounds somewhat comical and simplistic and uses a kazoo as well as incorporating various sound effects for which Pink Floyd would become renowned. 'Corporal Clegg had a wooden leg. He won it in the war in 1944'. Clegg's wooden leg is symbolic of his PTSD that he 'won' in the war. He is later reported to have found the leg 'in the zoo', which attests to the utter chaos of war.

'Were they really sad for me? Will they really laugh at me?', illustrates the complex feelings veterans often experience regarding their service, simultaneously questioning whether their civilian compatriots understand the degree of their sacrifice, whether they are being ridiculed for the mental health issues which they have developed or for having served in a conflict which many oppose. 'Mrs Clegg, you must be proud of him. Mrs Clegg, another drop of gin?', reminds us that for many sufferers of PTSD and their families substance misuse is a maladaptive and dysfunctional way of coping with their trauma and has been for centuries.

'Corporal Clegg, umbrella in the rain, he's never been the same', tells us that Clegg is depressed and profoundly scarred by his wartime experiences. Near the end of the track, 'Corporal Clegg received his medal in a dream', questions whether indeed he was even awarded a medal and whether his sacrifice was worthwhile. Later an officer shouts, 'Clegg! Been meaning to speak to you. About that leg of yours!', which could be viewed as reticence by commanding officers and top brass to address mental health issues, which alas still exists to this day.

Roger Waters' father was killed in action near Anzio in 1944, when Waters was only an infant. This, coupled with Barrett's demise, would serve as major influences in Waters' writing. Waters has been quite open about how his upbringing, including having an overbearing mother and not having a father, profoundly affected him and that he spent over 20 years in therapy trying to come to terms with these issues. 